\title{
Estrategia de Estado para el tratamiento de la atrofia muscular espinal: un desafío del sistema de salud en Argentina
}

State strategy for the treatment of spinal muscular atrophy: a challenge for the health system in Argentina

Estratégia de Estado para o tratamento da atrofia muscular espinhal: um desafio para o sistema de saúde da Argentina

Agustín Carignani ${ }^{1}$

Daniela Yannen Flores ${ }^{2}$

\section{Resumen}

Objetivo: analizar la implementación y comercialización de medicamentos de alto costo en el sistema de salud argentino, tomando como referencia el tratamiento médico de la enfermedad de atrofia muscular espinal y analizar la normativa aplicable, demonstrando el paralelismo legislativo con la República Federativa del Brasil. Metodología: se realizó una investigación descriptiva analítica identificando los criterios plasmados en las normas ó reglamentos para la implementación de una medicación de alto costo. Resultados: logramos obtener el marco regulatorio de la medicación denominada nusinersen en Argentina y la República Federativa del Brasil. Conclusión: con el avance de la tecnología, sus altos costos impactan directamente en la posibilidad de acceso, ante lo cual creemos que además de las herramientas de negociación interna de cada país, la cooperación internacional resulta una herramienta fundamental para transformar esta realidad en una oportunidad de acceso.

\section{Palabras clave}

Atrofia muscular espinal. Costos de los medicamentos. Derecho a la salud.

\begin{abstract}
Objective: to analyze the implementation and commercialization of high-cost drugs in the Argentine health system, taking as a reference the medical treatment of spinal muscular atrophy disease and analyze the applicable regulations, demonstrating the legislative parallelism with the Federative Republic of Brazil. Methods: a descriptive analytical investigation was conducted to identify the criteria set out in the rules or regulations for the implementation of a high-cost medication. Results: we obtained the regulatory framework for the medication called nusinersen in Argentina and the Federative Republic of Brazil. Conclusion: with the advancement of technology, its high costs have a direct impact on the possibility of access, before which we believe that in addition to the internal negotiation tools of each country, international cooperation is a fundamental tool to transform this reality into an opportunity access.

\section{Keywords}

Muscular atrophy spinal. Drug costs. Right to health.

\footnotetext{
${ }^{1}$ Doctorando, Facultad de Derecho y Ciencias Sociales, Universidad Nacional de Córdoba, Ciudad de Alta Gracia, Provincia de Córdoba, Argentina; magíster en Salud Pública, Escuela de Salud Pública, Facultad de Ciencias Médicas, Universidad Nacional de Córdoba, Ciudad de Córdoba, Provincia de Córdoba, Argentina. https://orcid.org/0000-0002-7126-9328. Email: agustincarignani@gmail.com.

${ }_{2}$ Abogada, Universidad Nacional de Córdoba, Ciudad de Córdoba, Provincia de Córdoba, Argentina; agente judicial, Cámara Federal de Apelaciones de Córdoba, Ciudad de Córdoba, Provincia de Córdoba, Argentina. https://orcid.org/00000002-0305-3517. E-mail: danielayflores@gmail.com.
} 


\section{Resumo}

Objetivo: analisar a implantação e comercialização de medicamentos de alto custo no sistema de saúde argentino, tomando como referência o tratamento médico da doença atrofia muscular espinhal; e analisar a regulamentação aplicável, demonstrando o paralelismo legislativo com a República Federativa do Brasil. Metodologia: foi realizada uma pesquisa descritiva analítica, identificando os critérios previstos nas normas ou regulamentos para a implantação de um medicamento de alto custo. Resultados: obtivemos o marco regulatório do medicamento denominado nusinersen na Argentina e na República Federativa do Brasil. Conclusão: os altos custos da tecnologia impactam diretamente no seu acesso. Acreditamos que, além dos instrumentos de negociação interna de cada país, a cooperação internacional é uma ferramenta fundamental para transformar essa realidade em oportunidade de acesso.

\section{Palavras-chave}

Atrofia muscular espinhal. Custos de medicamentos. Direito à saúde.

\section{Introducción}

Un sistema de salud es un modelo de organización social creado a los fines de dar respuesta a problemas de salud de la población. Específicamente en Argentina el sistema de salud está compuesto por el sector público: el Ministerio de Salud de la Nación, los ministerios provinciales y las secretarias de salud municipal; todos ellos con una red de hospitales con atención gratuita que pueden pertenecer a cualquiera de estas distintas órbitas. También existe un sistema privado compuesto de prestadores y empresas de medicina prepaga (1).

La Constitución Nacional Argentina (2) reconoce el derecho a la salud en sus artículos 14bis, 33, 42, 75 incisos 18 y 19, dejando claro que es un derecho del pueblo y como deber del Estado, a la vez que en virtud del art. 75 inc. 22 de dicho plexo legal, se incorpora una serie de tratados y convenciones internacionales tendientes también a su protección.

Brevemente, corresponde destacar que dentro del sistema sanitario argentino fue creada la Administración Nacional de Medicamentos, Alimentos y Tecnología Médica (ANMAT) como organismo descentralizado de la Administración Nacional de la República Argentina dependiente del Ministerio de Salud, con el objetivo de controlar y garantizar que los medicamentos, alimentos y dispositivos médicos posean eficacia, seguridad y calidad (3).

La lucha contra las inequidades, la aparición de nuevas enfermedades y la obligación del Estado argentino $^{3}(4)$ de hacer frente a la protección del derecho a la salud (5),

\footnotetext{
${ }^{3}$ Conforme lo hace Rosatti, hemos definido al Estado nacional como la "estructura organizativo- burocrática caracterizada por vincular institucionalmente a un territorio con una población que -más allá de sus disputas sectoriales- se considera contenida por un orden jurídico relativamente hermenéutico y expresada por intermedio de símbolos apropiados, gozando tales características (pertenencia territorial, identidad nacionalista, juridicidad propia, capacidad organizativa) del
} 
impulsaron la intervención activa de distintas órbitas del Poder Ejecutivo Nacional en materia de salud, a los fines de garantizar el acceso de todos los habitantes.

El Estado argentino por su carácter de institución compleja, de fines amplios, variados y variables, permite organizar la convivencia social y regular conflictos, requiriendo de recursos para su buen funcionamiento el cual no queda librado a sí mismo, sino al control social (6). Por ello, una política clara sobre salud resulta fundamental para fortalecer el acceso de toda la población.

Las nuevas enfermedades que no poseen tratamiento científico aprobado (experimentales) o que poseen un alto costo económico, o que por distintas razones se encuentran dentro del nomenclador nacional, entre otras, vulneran el acceso igualitario al sistema sanitario, pues no todos pueden acceder, llegando a hacerlo sólo quienes accedan por medio de los órganos judiciales

Este trabajo tiene como objetivo analizar las dificultades de la implementación y comercialización de medicamentos de alto costo en el sistema de salud argentino, tomando como referencia el caso del tratamiento médico (medicinal) para la enfermedad atrofia muscular espinal y analizar la normativa aplicable, como así también, mostrar el paralelismo legislativo con la República Federativa del Brasil.

\section{Metodología}

En el presente trabajo realizamos una investigación descriptiva, analítica, identificando los criterios plasmados en las normas y reglamentos para la implementación de una medicación de alto costo en el nomenclador nacional.

La medicación analizada en poco tiempo generó una proliferación normativa (vía reglamentos o infraconstitucionales) muy grande, existiendo incluso normas que, al poco tiempo de ser dictadas, fueron derogadas. Al estudiarlas, advertimos referencias a procedimientos llevados adelante por la República Federativa del Brasil para la atención de esta medicación que llamaron nuestra atención.

\section{La atrofia muscular espinal (AME) y su tratamiento}

Conforme la identifica el National Institute of Neurological Disorders, la Atrofia Muscular Espinal (AME) es una enfermedad genética, hereditaria (causada por defectos en

reconocimiento internacional" (4). 
el Gen SMN1), degenerativa que ataca las células nerviosas llamadas neuronas motoras que se encuentran en la médula espinal, altamente discapacitante, definida como poco frecuente y con elevada mortalidad en sus formas más graves, clasificándose en cuatro grupos sobre la base de la gravedad de los síntomas, la edad de aparición y la evolución: AME I, II, III (A-B) y AME IV (7).

Si bien se han desarrollado en los últimos años nuevas tecnologías para el tratamiento de esta enfermedad genética ${ }^{4}(8)$, centraremos el análisis en la medicación desarrollada por el Laboratorio Biogen, denominado nusinersen y cuyo nombre comercial es Spinraza $\AA^{\circledR}$, por ser la única aprobada en nuestro país hasta el momento por la Administración Nacional de Medicamentos, Alimentos y Tecnología (ANMAT), a la vez que su inclusión dentro del nomenclador prestacional nacional exterioriza similares características a las desarrolladas en países de la región. En definitiva, la inclusión de esta medicación, y su evolución y regulación normativa, nos posiciona frente a un caso paradigmático.

El 19.09.2018 la Honorable Cámara de Diputados de la Nación requirió al Poder Ejecutivo, vía pedido de informe la cantidad de pacientes que estaban bajo tratamiento para AME y cuántos pacientes tenían registrados con esta patología, pese a que dicho informe afirmaba que había trescientas (300) familias con pacientes que sufrían la patología. La importancia del tema radica en el complejo acceso que se tiene a dicha medicación, por ser de alto costo destinada al tratamiento de una enfermedad poco frecuente, que cuenta con resultados científicos positivos en pacientes que la han utilizado, y por justamente tratarse de una medicación que ha traído múltiples discusiones (9) sobre su incorporación en el nomenclador nacional.

$\mathrm{Si}$ bien la medicación es relativamente nueva, la misma fue aprobada por la Administración de Medicamentos y Alimentos (FDA) (10) en Estados Unidos en el año 2016 y en Europa en el año 2017, resultando hoy en día la esperanza de los pacientes y sus familias que deben transitar por esta enfermedad (11).

Observamos que desde un marco legal que respeta el terreno de la ética y la justicia distributiva, se proyecta un camino que busca unificar las obligaciones asumidas por el Estado Nacional Argentino en materia de salud, con las posibilidades económicas de los agentes de salud y del propio Estado, de tal manera que su acceso no sea necesario a través del sistema judicial.

\footnotetext{
${ }^{4}$ En la actualidad se encuentra aprobado por la FDA la medicación para el tratamiento de AME denominada Zolgensmaß desarrollada por el laboratorio Novartis y por otro lado, se encuentra bajo estudio, la medicación EVRYSDI ${ }^{\circledR}$ (risdiplam) del laboratorio Roche (la primera que puede ser tomada oralmente).
} 


\section{Justicia e Igualdad}

En palabras de Aristóteles (12)

Consistiendo la justicia en lo justo, en lo igual y en un cierto medio, lo justo solo puede ser lo justo entre ciertos seres, lo igual no puede ser igual sino para ciertas cosas, y el medio solo puede ser el medio también entre ciertas cosas. De aquí se deduce que la justicia y lo justo son relativos a ciertos seres y a ciertas cosas.

Estamos frente a un ordenamiento jurídico que reconoce a la vida como fundamento de todos los demás derechos (13), encontrando al Estado Nacional como garante de este ejercicio frente a la necesidad de lograr que la cobertura de salud también asegure un desarrollo sustentable - conforme se afianzó en la Organización de las Naciones Unidas (14) en su Objetivo № 3 (particularmente punto 3.8) -, y el acceso y cobertura de esta medicación ha presentado frondosas discusiones desde el punto de vista ético, moral y jurídico.

Dichas discusiones terminan muchas veces dilucidándose en la justicia, cuando se reclama una necesidad de salud insatisfecha, donde la persona se encuentra muchas veces ante una doble injusticia; por un lado, la falta de reconocimiento de un derecho innato y por el otro, la necesidad de recurrir a un órgano no natural que resuelva el conflicto.

Si bien entendemos que no debiera ser necesaria la mediación de los organismos judiciales para resolver derechos sociales insatisfechos (15) (específicamente el de la salud), ante la necesidad de los miembros de la sociedad de acceder a un tratamiento que asegure su vida o su derecho a la salud, resulta inevitable que los afectados inicien los respectivos reclamos judiciales para obtener la respuesta a su reclamo, lo que sólo puede ser zanjado, inevitablemente - y valga la redundancia -, mediante un reclamo judicial. Y aquí es donde podemos ver lo que anteriormente mencionamos como lo justo entre ciertos seres, ya que en definitiva, la persona que debe recurrir al órgano jurisdiccional, además de ver conculcado su derecho a la salud, se ve afectado por tener que recurrir a la justicia para obtener una respuesta.

Al respecto, están quienes entienden como Corvalan (16) que

[...] una vez que se han garantizado pisos mínimos, el Poder Judicial no puede decidir (interpretar y ponderar) si la implementación de las políticas públicas que balancean la ecuación derechos-prioridades-obligacionesrecursos, está o no optimizada con relación a un derecho concreto (salud, vivienda, etcétera). 
Sin embargo, tal como se aseguró antes, encontrando en la vida el mayor valor de todo el ordenamiento jurídico y ante la falta de previsión para una determinada tecnología médica, el último recurso del paciente es el acceso a la justicia.

En definitiva, la necesidad de recurrir al Poder Judicial para el reconocimiento de un derecho es un hecho, el cual no garantiza igualdad para todos aquellos que requieren una cobertura de salud. Asimismo, observamos que ante la judicialización de la salud por la vía del amparo ó bioamparos (17) - vía utilizada para el reclamo de prestaciones de estas características - trae aparejado además un desgaste jurisdiccional (evitable) y un alto costo para quienes deciden interponer dicha acción. Este acceso a la justicia para resolver estos planteos no es nuevo y viene acompañado del exponencial crecimiento de este tipo de acciones judiciales lo que ha sido advertido en reiteradas oportunidades. El Prof. Dr. Carnota (18) sostuvo que

\footnotetext{
(I)os nuevos constitucionalismos impulsaron la simplista visión de que el mero reconocimiento de una facultad pública subjetiva en el marco de un documento constitucional e internacional bastaba para conferir legitimidad, y lo que es más importante desde un ángulo práctico, operatividad, para exigir el cumplimiento de determinadas prestaciones.
}

En una batalla contra el tiempo, en búsqueda de soluciones médicas que sirvan de respuesta a la enfermedad y bajo la necesidad de encontrar un reparo donde a veces ni las instituciones médicas ni el Estado lo tienen, la justicia viene a ser la vía idónea en circunstancias excepcionales para brindar las respuestas en base al principio de igualdad ante la ley, aunque muchas veces, sin las herramientas suficientes que den la tranquilidad al sentenciante más que la ciencia acompañada en autos.

\section{Marco legal: evolución de la normativa argentina}

Para lograr entender la situación actual es necesario desandar el marco legal. En el año 1996 se constituyó la Superintendencia de Servicios de Salud como organismo descentralizado de la Administración Pública Nacional (19) con el objetivo de implementar, reglamentar y administrar los recursos provenientes del Fondo Solidario de Redistribución, dirigiendo todo su accionar al fortalecimiento cabal de la atención de la salud de los beneficiarios del Sistema Nacional del Seguro de Salud, destinando los recursos disponibles para la cobertura de reintegros por prestaciones de alto impacto económico y que demanden una cobertura prolongada en el tiempo, a fin de asegurar el otorgamiento de prestaciones 
de salud igualitarias, garantizando a los beneficiarios la obtención del mismo tipo y nivel de prestaciones.

En el año 2012 con la sanción del Decreto № 2710/2012, se prevé el otorgamiento de prestaciones de salud debiendo utilizar racionalmente los recursos y fondos disponibles (20). También se dictó la Disposición № 4622 (21) para evaluar el registro de enfermedades poco frecuentes bajo condiciones especiales.

Al determinar que la Superintendencia de Servicios de Salud tiene también como objetivo que los beneficiarios del Seguro de Salud tengan un acceso equitativo a los recurso tecnológicos que requieran para el tratamiento de sus enfermedades o dolencias que padecen, y en el marco de mejorar la calidad de atención, optimizando los recursos disponibles y dando dinámica y transparencia a la gestión institucional, se reformuló el Sistema Único de Reintegros mediante la Resolución № 1200/12 (22); donde se debe acreditar el efectivo pago de la prestación a reintegrar y su cumplimiento efectivo.

Mediante la Resolución № 400/16 (23) (modificado por Res. № 46/17) la Superintendencia de Servicios de Salud aprobó los requisitos generales, específicos, coberturas, medicamentos y valores máximos a reintegrar a los agentes de salud en el marco del Sistema Único de Reintegros.

Mediante la Ley № 16.463 (24) se estableció la autorización a la importación, exportación, producción, elaboración, fraccionamientos, comercialización o depósito en jurisdicción nacional o con destino al comercio interprovincial de las drogas, productos químicos, reactivos, formas farmacéuticas, medicamentos, elementos de diagnóstico y todo producto de uso y aplicación en medicina humana y que se deben realizar previa autorización y control de la autoridad sanitaria correspondiente (art. $1^{\circ}$ y $2^{\circ}$ ), estableciéndose además un procedimiento operativo uniforme y con respaldo científico.

Mediante la Resolución № 623/2018 (25) se creó la Comisión Nacional de Evaluación de Tecnologías Sanitarias (Conetec) y se estableció en su artículo 6으 las competencias de este organismo a los fines de evaluar productos médicos, instrumentos, técnicas y procedimientos clínicos y quirúrgicos destinados a la prevención, diagnóstico y tratamiento de enfermedades o rehabilitación de salud a fin de determinar su uso apropiado, oportunidad y modo de incorporación.

La Conetec, elaboró un informe ${ }^{5}$ (26) sobre la droga nusinersen - dictamen que fue luego acogido por las nuevas normas reguladoras - en el cual afirman que en caso de

${ }^{5}$ Corresponde remarcar que este informe ha sido refutado por el laboratorio Biogen, conforme las razones expuestas en 
reducción considerablemente significativa en el precio de la tecnología que permita garantizar la sustentabilidad del resto de las prestaciones esenciales como promoción de salud, prevención, tratamiento y rehabilitación para todos los beneficiarios del sistema de salud argentino, podría considerarse la cobertura para pacientes con AME tipos I y II. Asimismo, se sostuvo que se debía incluir la nueva tecnología dentro del Sistema de Tutelaje de Tecnologías Sanitarias Emergentes de la Superintendencia de Servicios de Salud con el objetivo de poder realizar un seguimiento y análisis de su eficacia y seguridad atento a poseer beneficio clínico para pacientes con la enfermedad.

El Laboratorio Biogen Argentina S.R.L. solicitó la inscripción en el Registro de Especialidades Medicinales (REM) de la Administración Nacional de Medicamentos, Alimentos y Tecnología Médica (ANMAT) de la droga nusinersen, a lo fines de la importación para la República Argentina, autorizándose bajo condiciones especiales por el plazo de un año conforme la Disposición № 2062/2019 (27).

Con fecha 2 de mayo de 2019 se celebró un acuerdo entre la Secretaría de Gobierno de Salud del Ex Ministerio de Salud y Desarrollo Social y Biogen Argentina S.R.L. mediante el cual se preveía el suministro y comercialización del medicamento con nombre comercial Spinraza $\AA$ a un valor inferior al de plaza (28).

Mediante la Resolución № 1114/2020 (29), la autoridad de aplicación valoró la situación de la medicación y solicitó a la Secretaría de Comercio Interior del Ministerio de Desarrollo Productivo su colaboración para garantizar la provisión del medicamento Spinraza ${ }^{\circledR}$ en el mercado argentino a un precio aún inferior al hasta entonces convenido, dando por concluido el contrato firmado con el laboratorio en el año 2019, en función de la promoción de un uso racional de las tecnologías y de una eficiente distribución de los recursos del sistema de salud.

Posteriormente, conforme Disposición № 4529/20 (30), y de acuerdo a la evidencia científica recabada durante más de un año en el uso del producto, se reinscribe el certificado correspondiente a Spinraza $\AA^{\circledR}$ por el término de un año, bajo exclusiva indicación médica para realizar el tratamiento de atrofia muscular espinal tipos I y II (dejando fuera del registro AME III), bajo la modalidad de cobertura que recomiende la Conetec.

En este marco de nuevas políticas del Estado Nacional, se dicta la Resolución № 1115/2020 (31) por medio de la cual se deroga la Resolución № 1452/19 por la que se había incluído nusinersen al Programa Médico Obligatorio (PMO) para el tratamiento de AME tipos 
I, II y IIIA, al entender que el alto costo de la medicación, "[...] tendría un impacto negativo sobre la salud pública, la equidad y en el aspecto económico [...]". Al respecto, hallamos importante destacar que al haberse incluido en el PMO la medicación - entendiéndolo como un conjunto de prestaciones médicas básicas que los agentes de seguro de salud deben garantizar a sus beneficiarios - los agentes de salud se encontraban obligados a brindar la cobertura total de esta medicación; y que si bien por medio de la resolución № 1115/2020 se la excluye del mismo, se deja en claro que los agentes de salud deben continuar brindando la cobertura a aquellos pacientes con AME que ya habían iniciado el tratamiento con la medicación durante el periodo de validez de la Resolución № 1452/19.

Por su parte, la Superintendencia de Servicios de Salud dictó la Resolución № 597/2020 (32) e incorpora la medicación al Sistema de Tutelaje de Tecnologías Sanitarias Emergentes a los fines de asegurar una racional y eficiente distribución de los recursos afectados por el Fondo Solidario de Redistribución (SUR), y sólo para AME tipos I y II en consonancia con la Disposición № 4529, lo que conforme afirma dicha normativa, permitirá contar con datos clínicos más efectivos respecto a la evolución de la enfermedad como así también respecto de la respuesta a tratamientos, aportando datos para el correcto seguimiento de los pacientes alcanzados, velando por el efectivo otorgamiento de coberturas médicas pertinentes, adecuadas, seguras y de calidad, como así también se la incorpora a la Resolución № 400/16 de la Superintendencia de Servicios de Salud.

Bajo el entendimiento que el Estado Nacional debe garantizar derechos esenciales a la población y su efectivo goce, previendo que el art. 42 de la Constitución Nacional Argentina (33) establece que los consumidores y usuarios de bienes y servicios tienen derecho a la protección de su salud, seguridad e intereses económicos, a una información adecuada y veraz, a la libertad de elección y a condiciones de trato equitativo y digno el Ministerio de Desarrollo Productivo de la Secretaría de Comercio Interior dictó la Resolución № 202/2020 (34), mediante la cual establece como precio máximo de venta el principio activo nusinersen (Spinraza®) 12mg/5 ML por un vial, al valor de dólares estadounidenses veintisiete mil (U\$S 27.000). Asimismo, encomienda a la Comisión Nacional de Defensa de la Competencia la realización de la investigación de mercado para poder determinar la posibilidad de comisión de infracciones a la Ley de Defensa de la Competencia № 27.442.

Por último, con fecha 11.11.2020 fue sancionada la Resolución № 1860/2020 (35) mediante la cual se crea la Comisión Nacional para Pacientes con Atrofia Muscular Espinal, en la órbita del Programa Nacional de Enfermedades Poco Frecuentes, la que funcionará 
conforme a lo establecido en el Reglamento de Organización y Funcionamiento y se sujetará a las Pautas para la Cobertura de Nusinersen a Pacientes con Atrofia Muscular Espinal, que se adjuntan como Anexos I (IF-2020-72264996-APN-DCAP\#MS) y II (IF-2020-59361579APN-DCAP\#MS) respectivamente, de la Resolución.

De la normativa citada, queda acreditada la mutación normativa que existió entre los años 2019 y 2020 en el tratamiento de un medicamento con las características de nusinersen, y los distintos órganos de gobierno que debieron interactuar para ello.

\section{Derecho Comparado: la situación de Brasil y la aceptación de la comercialización de nusinersen (Spinraza ${ }^{\circledR}$ )}

Conforme hemos podido analizar para este trabajo, las discusiones en torno a la incorporación de nusinersen en el nomenclador de medicamentos en distintos países del mundo, y particularmente en los de la región, ha generado análogos ejes temáticos que orbitan básicamente en torno a la efectividad del medicamento y fundamentalmente su costo.

Es notoria la similitud del tratamiento en la República Federativa de Brasil y sin duda alguna, esta similitud responde a la proximidad de las características del Sistema Público de Salud argentino con el Sistema Único de Salud brasilero, en el marco de los cuales, se presentan discusiones o dilemas relacionados con la concretización del derecho a la salud, su acceso y los desafíos que presentan estos sistemas garantistas en miras de la universalidad equitativa-distributiva y solidaria frente a una realidad de perogrullo que es la finitud de los recursos.

Brasil ha reconocido también a nusinersen como único tratamiento para pacientes con AME. Con fecha 8 de enero de 2018 la agencia Nacional de Vigilancia Sanitaria de Brasil (Anvisa) mediante la Resolución RDC № 202/2018 (36), eliminó algunos requisitos que se exigían con anterioridad, a los fines de simplificar los procedimientos en busca de tener un impacto positivo en el costo de almacenamiento de las empresas que traen productos relacionados con la salud para ese país, retirando concretamente las exigencias de documentos que las empresas solo conseguían después que las cargas llegaban al país, lo que generaba costos de almacenamiento y hacía aumentar el precio final de los productos.

Mediante la Portaria № 24 de fecha 24 de abril de 2019 (37), se incorporó el medicamento Spinraza ${ }^{\circledR}$ al sistema sanitario brasilero para aquellos pacientes con diagnóstico de AME tipo I de acuerdo a lo recomendado por la Comisión Nacional de Incorporación de Tecnologías SUS (Conitec). Luego, mediante la Portaría Conjunta № 15 
de fecha 22 de octubre de 2019 se aprueba el Protocolo Clínico y de Directrices Terapéuticas para el tratamiento de la atrofia muscular espinal $5 q$ tipo I (38).

Dicho esto, mediante el dictado de las últimas dos ordenanzas, se observa que la decisión del Ministerio de Salud brasilero activa un mecanismo de control a nivel nacional al establecer que el protocolo para AME $5 q$ tipo I, los criterios diagnósticos, criterios de inclusión y exclusión, tratamientos y regulacion, mecanismos de control y evaluación ${ }^{6}$, es de carácter nacional y debe ser utilizado por los Departamentos de Salud de los Estados, el Distrito Federal y los Municipios en la regulación de acceso a la asistencia, autorización, registro y reembolso de los trámites correspondientes.

Sin embargo, considerando que no fueron incorporados los pacientes con AME tipos II y III y teniendo en cuenta la evidencia científica que atestiguaba la eficacia y seguridad del tratamiento con Spinraza $\AA^{8}$ para pacientes con estos grados de enfermedad, mediante la Portaria № 1297 del 11 de junio de 2019 (39) se estableció un proyecto piloto instituido como convenio de riesgo compartido para la incorporación de tecnologías sanitarias, con el fin de ofrecer el acceso al medicamento Spinraza ${ }^{\circledR}$ para el tratamiento de AME tipos II y III en el ámbito del Sistema Único de Salud (SUS). Conforme el art. $1^{\circ}$ de dicha norma se incorpora la autorización del tratamiento para los tipos de AME II y III, al encontrar como fundamento la necesidad de orientar y coordinar acciones específicas sobre un convenio de riesgo compartido entre el Ministerio de Salud y la empresa farmacéutica que suministra el medicamento, ofreciendo así acceso al tratamiento de AME tipos II y III en el ámbito del Sistema Único de Salud (SUS). Dicho convenio se suscribió teniendo en cuenta el costo y efectividad del medicamento, la estimación del consumo, considerando la calidad de dosis y el impacto presupuestario, todo en miras de posibilitar la incorporación del medicamento para los AME II y III en el ámbito del SUS. Se tuvo en vista también, promover el balance del costo del vial, recopilar evidencia adicional sobre el uso del medicamento en condiciones reales, posibilitar reevaluar la incorporación de la tecnología ante evidencias adicionales, subsidiar la publicación de una futura norma general sobre el acuerdo de riesgo compartido para la incorporación de tecnologías sanitarias y, por último, promover la investigación en atrofia muscular tipos II y III para orientar políticas de salud pública (40).

Asimismo, se establece cómo será el reparto de riesgos el que deberá estar debidamente motivado por el comunicado del Departamento de Ciencia, Tecnología, Innovación y Suministros Estratégicos de Salud (SCTIE/MS) presentando las bases técnicas

\footnotetext{
${ }^{6} \mathrm{http}: / /$ portalms. saude.gov.br/protocolos-e-diruntas
} 
de criterios utilizados. Por otro lado, establece que los pacientes tendrán acceso al medicamento en los centros de referencia para el tratamiento de AME, los cuales serán seleccionados por el Servicio de Referencia en Enfermedades Raras y los Hospitales Universitarios Estatales y Federales.

Continuando con este análisis, conforme da cuenta la doctrina internacional (39), el acuerdo de riesgo compartido entre Brasil y la empresa farmacéutica, radica en la reducción del precio de los medicamentos, la descripción de la enfermedad y los criterios de elegibilidad de los subgrupos de pacientes que se beneficien de acuerdo al riesgo, la definición de los criterios de resultados de salud esperados y los parámetros de efectividad clínica, el número máximo de pacientes por año que recibirán la tecnología a cargo del Ministerio de Salud en base a criterios epidemiológicos y/o estimación de la demanda con la previsión de que si se supera este número, la empresa farmacéutica correrá con el costo del medicamento para los otros pacientes, la definición de los criterios para interrumpir el suministro de la medicación en los pacientes que no presentan los resultados de salud esperados y la periodicidad con la que evaluará la clínica de los pacientes.

En efecto, el tratamiento de este tipo de enfermedades de alto costo, incapacitantes, encuadradas como enfermedades poco frecuentes ó huérfanas, llevan al replanteo de la afectación de recursos para un tratamiento equitativo y para el mejor tratamiento de la enfermedad y a su vez, para garantizar el sostenimiento del sistema de salud en su integridad.

De la recapitulación hecha de la situación en nuestro país vecino, vemos cómo las dificultades y objetivos son idénticas. Es decir, en sistemas de salud análogos, los ejes de contacto son palmarios. Evidencia este diálogo de las distintas jurisdicciones cuando la Resolución № 202/2020 (41) del Ministerio de Desarrollo de la Nación de Argentina, advierte que el precio que estaba acordado entre Argentina y el Laboratorio productor del medicamento era sensiblemente superior al que estaba acordado en "[...] al menos, otro país de la región, como resulta del informe acompañado por el MINISTERIO DE SALUD, para el caso de la REPÚBLICA FEDERATIVA DEL BRASIL"7, razón por la cual, se ajustó dicho monto a la suma de dólares estadounidenses veintisiete mil (U\$27.000).

Asimismo, observamos que otra distincion, además del costo, es que en Argentina se prevé el tratamiento con esta medicación para los pacientes con AME tipos I y II, mientras que en Brasil sólo se encuentra autorizado el protocolo para aquellos que padezcan AME

${ }^{7}$ Conforme los considerandos de la Resolución № 202/20 del Ministerio de Desarrollo Productivo de la Nación. 
tipo I, sin incorporar en la Ordenanza № 24 los subtipos II y III, lo que motiva el dictado de la Ordenanza № 1297 que denomina como proyecto piloto de convenio de riesgo compartido la incorporación de esta tecnología para dichos subtipos de AME. Esta regulación normativa, permite poder estudiar con mayor precaución el impacto de la medicación a los pacientes con esos subtipos de la enfermedad, al entender las autoridades brasileras que si bien existe

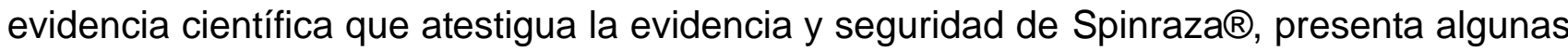
incertidumbres acerca del número de pacientes evaluados.

Tal como ha sucedido en otras oportunidades ${ }^{8}$, Argentina a los fines de garantizar el adecuado cumplimiento de los Tratados Internacionales de los cuales es parte, miró a sus países vecinos para tener como referencia el modo en que fue resuelta la situación relativa a esta medicación.

Y en efecto, es que no existe discusión en términos generales sobre la obligación de cobertura de salud a cargo de los distintos agentes de salud e incluso del Estado Nacional en su carácter de garante del sistema de salud; sin embargo, ello no excluye la difícil discusión de la afectación de recursos, y es que la sabida finitud de recursos, obliga a su aplicación dentro del esquema de eficacia.

\section{Conclusiones}

Ocurre que los gastos en salud son crecientes, los medicamentos y tecnologías se renuevan a una velocidad inusitada que no permiten evaluarlas con los actuales parámetros existentes que permitan dotarlos de la confiabilidad que se requiere para su implementación y comercialización.

En este orden de ideas, teniendo en cuenta que - en el caso particular - el producto nusinersen es el único aprobado por ANMAT, ello da lugar a un mercado nominado, concentrado y monopolizado por el Laboratorio Biogen, por lo cual, bajo la óptica de que los consumidores tienen derechos constitucionales para la protección de su salud y su seguridad, las autoridades deben defenderlos contra toda forma de distorsión del mercado pudiendo así, controlar los monopolios, garantizando los derechos esenciales a la población y su goce efectivo.

\footnotetext{
${ }^{8}$ Ejemplo de ello lo encontramos años atrás con el trasplante de pulmón entre donantes vivos, práctica que hasta el año 2012 no estaba prevista en la legislación argentina, pero sí estaba en la legislación brasilera. Caso concreto lo vemos en las acciones judiciales entabladas en contra del Estado Nacional, el Estado Provincial (Córdoba) y la Administración Provincial del Seguro de Salud (42).
} 
En estos sistemas de salud pública, como el de Argentina y Brasil, no hay duda alguna que es deber del Estado la garantía del acceso al sistema de salud. Ello por cuanto la garantía del disfrute del derecho a la salud por medio del órgano judicial, si bien asegura aquel derecho en favor de un ciudadano, jamás puede constituir una política pública; y en tal sentido resulta fundamental arbitrar los medios que resulten necesarios para generar políticas integrales, sustentables y equitativas que garanticen la subsistencia del sistema público de salud, sin que los particulares deban judicializar sus requerimientos.

Ante el evidente incremento de costos en nuevas tecnologías, en el marco de una limitada capacidad económica y técnica que permita despejar toda duda sobre la eficacia de las nuevas tecnologías, es claro que, o por acuerdo de riesgo compartido, o por la directa e inmediata intervención del Estado en la regulación de precios, existen herramientas que ayudan a afrontar estos nuevos costos y paliar de alguna manera la necesidad de llegar al órgano judicial para asegurar la garantía del derecho constitucional elemental, que es la vida y su correlativo derecho a la salud; ello, en el marco de las características propias de nuestros sistemas de salud.

Por ello, observamos que ante esta necesidad, puede surgir la oportunidad - ¿por qué no? - de tener que implementar políticas coordinadas entre los países de la región y afectados por una misma necesidad como estrategia destinada a fortalecer el desarrollo económico y social, promoviendo fuerzas tendientes a integrar un mismo espacio regional entre países geográficamente próximos y económicamente complementarios.

Observamos que en un mundo globalizado, donde las economías son dinámicas y competitivas, posicionados frente a sistemas sanitarios análogos, el poder de negociación y cooperación entre los países de cada región en casos tan emblemáticos como complejos, luciría como respuesta y estrategia para reforzar el bloque comercial.

En este marco de ideas, estamos convencidos que una política sanitaria sólida, estable y dinámica, donde las herramientas y el camino para el acceso a los servicios de salud estén claros, las actuaciones judiciales ante los organismos jurisdiccionales disminuirían considerablemente, lo que en definitiva, repercutirá positivamente en los principios rectores que constituyen al sistema público de salud.

\section{Referencias}

1. Carignani A, Robledo F. El Sistema de Protección de la Salud de la Argentina: perspectivas y desafíos. Cad. Ibero-am. Dir. Sanit. [Internet]. 2015 [consultado el 24.11.2020];8(4):143-150. Disponible en: 
https://www.cadernos.prodisa.fiocruz.br/index.php/cadernos/article/view/186/219. doi: https://doi.org/10.17566/ciads.v4i2.186

2. Argentina. Constitución Nacional, de 15 de diciembre de 1994. Senado y Cámara de Diputados de la Nación Argentina. Boletín Oficial; 3 de enero de 1995.

3. Argentina. Ministerio de Salud de la Nación. Decreto № 1490/92, de 20 de agosto de 1992. Declaración de interés nacional [Internet]. Boletín Oficial; 27 de agosto de 1992 [consultado el 29.11.2020]. Disponible en:

http://www.anmat.gov.ar/webanmat/Legislacion/NormasGenerales/Decreto_1490-1992.pdf

4. Rosatti HD. La Construcción del Estado Argentino: ensayos de historia argentina. Santa Fe:Rubinzal-Culzoni Editores; 1994. p. 9.

5. Donato NA. Derecho a la salud [Internet]. Diccionario Enciclopédico de la Legislación Sanitaria Argentina. 2017 [consultado el 24.11.2020]. Disponible en:

http://www.salud.gob.ar/dels/printpdf/135

6. Rosatti HD. Tratado de Derecho Constitucional. 2o Edición. Santa Fe:Rubinzal-Culzoni Editores; 2017. p. 29-30.

7. National Institute of Neurological Disorders and Stroke [Internet]. Atrofia muscular espinal. 2016 [consultado el 18.11.2020]. Disponible en:

https://espanol.ninds.nih.gov/trastornos/atrofia-muscular-espinal.htm

8. FDA [Internet]. FDA Approves Oral Treatment for Spinal Muscular Atrophy [Internet]. 07 agosto 2020 [consultado el 29.11.2020]. Disponible en https://www.fda.gov/news-

events/press-announcements/fda-approves-oral-treatment-spinal-muscular-atrophy

9. Cámara de Diputados de Argentina. Proyecto de Resolución del 05/09/2018. Pedido de informes al poder ejecutivo sobre el registro de pacientes con "atrofia de medula espinal ame -", y otras cuestiones conexas. [consultado el 25.11.2020]. Disponible en: https://www.diputados.gov.ar/proyectos/proyecto.jsp?exp=5496-D-2018

10. Agencia Europea de Medicina [Internet]. Spinraza - Resumen del EPAR para el público general. 2017 [consultada el 27.11.2020]. Disponible en:

https://www.ema.europa.eu/en/documents/overview/spinraza-epar-summary-public_es.pdf

11. FDA [Internet]. FDA approves first drug for spinal muscular atrophy [Internet]. 23 dezembro 2016 [consultada el 27.11.2020]. Disponible en: https://www.fda.gov/newsevents/press-announcements/fda-approves-first-drug-spinal-muscular-atrophy

12. Aristóteles. La Gran Moral [Internet]. Capítulo XXXI, De la Justicia. En: Azcárate P de. Obras de Aristóteles. Proyecto Filosofía en español. 2005 [consultado el 19.11.2020]. Disponible en: http://www.filosofia.org/cla/ari/azc02045.htm

13. Chomali F. Derecho a la vida, derecho fundamental. Teología y Vida [Internet]. 2007 [consultado el 16.11.2020];48(4):413-423. Disponible en:

https://scielo.conicyt.cl/scielo.php?script=sci_arttext\&pid=S0049-34492007000300005 
14. Naciones Unidas [Internet]. Objetivo 3: Garantizar una vida sana y promover el bienestar para todos en todas las edades. Disponible en: https://www.un.org/sustainabledevelopment/es/health/

15. Caso Cuscul Pivaral y otros vs. Guatemala (2018). Corte Interamericana de Derechos Humanos. [consultado el 30.11.2020]. Disponible en:

https://www.corteidh.or.cr/docs/casos/articulos/seriec_359_esp.pdf

16. Corvalan GJ. Derecho administrativo en transición. Buenos Aires: Astrea; 2016. p. 278.

17. Carnota WF. El bioamparo como objeto del Derecho Procesal Constitucional. En: Ferrer EMG y otro. La ciencia del Derecho Procesal Constitucional. México: UNAM y Marcial Pons; 2008. p. 181.

18. Carnota WF. Limitaciones razonables al derecho a las prestaciones médicas. El Dial [Internet]; 14.06.2017 [consultada el 24.11.2020]. Disponible en:

https://www.eldial.com/nuevo/lite-tcd-

detalle.asp?id=9103\&base=50\&id_publicar=\&fecha_publicar=14/06/2017\&indice=doctrina\& suple=Publico

19. Argentina. Poder Ejecutivo Nacional. Superintendencia de Servicios de Salud. Decreto № 1615/1996, por el cual se fusiona la ANSSAL al INOS y al DINOS para constituir la Superintendencia de Servicios de Salud. 31 de diciembre de 1996.

20. Argentina. Poder Ejecutivo Nacional. Superintendencia de Servicios de Salud. Decreto № 2710/2012 de 28 de diciembre de 2012 y publicada en el B.O. el 17.01.2013.

21. Argentina. Ministerio de Salud de la Nación (ANMAT). Disposición № 4622/2012 de fecha 07 de agosto de 2012 [consultado el 20.11.2020]. Disponible en:

http://www.anmat.gov.ar/boletin_anmat/agosto_2012/Dispo_4622-12.pdf

22. Argentina. Superintendencia de Servicios de Salud. Resolución № 1200/2012 de fecha 21 de septiembre de 2012 por el que se crea el Sistema Único de Reintegros. [consultado el 26.11.2020]. Disponible en: http://servicios.infoleg.gob.ar/infoleglnternet/anexos/200000204999/202779/norma.htm

23. Argentina. Ministerio de Salud de la Nación. Resolución № 400/16 de fecha 25 de octubre de 2016 por el cual se establece el máximo a restituir por medicamentos.

[consultado el 26.11.2020]. Disponible en:

http://servicios.infoleg.gob.ar/infolegInternet/anexos/265000-269999/266970/norma.htm

24. Argentina. Ley № 16.463 del 23 de julio de 1964. Congreso de la Nación. Establece los requisitos de exportación, importación, comercialización, etc. de los medicamentos. [consultado el 26.11.2020]. Disponible en: http://servicios.infoleg.gob.ar/infoleglnternet/anexos/20000-24999/20414/norma.htm 
25. Argentina. Ministerio de Salud de la Nación. Resolución № 623/2018 de fecha 27.03.2018. [consultado el 29.11.2020]. Disponible en:

https://www.argentina.gob.ar/sites/default/files/resolucion_623-18.pdf

26. Comisión Nacional de Evaluación de Tecnologías de Salud - Conetec. Nusinersen en atrofia muscular espinal [Internet]. Informe de Evaluación de Tecnologías Sanitarias, №01. noviembre 2019 [consultado el 16.11.2020] Disponible en:

http://iah.salud.gob.ar/doc/428.pdf

27. Argentina. Administración Nacional de Medicamentos, Alimentos y Tecnología Médica. Disposición № 2062 de fecha 01 de marzo de 2019 por medio de la cual se inscribió en el Registro de Especialidades Médicas (REM) y "bajo condiciones especiales" a Nusinersen de Laboratorio Biogen. [consultado el 29.11.2020]. Disponible en: http://www.anmat.gov.ar/boletin_anmat/marzo_2019/Dispo_MSYDS_2062-19.pdf

28. Argentina [Internet]. Argentina: Ministerio de Salud. El Estado nacional logró un acuerdo innovador para garantizar el acceso al tratamiento de la Atrofia Muscular Espinal (AME); 08 de mayo de 2019 [consultado el 25.11.2020]; [Ministerio de Salud]. Disponible en: https://www.argentina.gob.ar/noticias/el-estado-nacional-logro-un-acuerdo-innovadorpara-garantizar-el-acceso-al-tratamiento-de

29. Argentina. Ministerio de Salud de la Nación. Resolución № 1114 de fecha 26 de junio de 2020 por medio de la cual se rescinde el Acuerdo de fecha 2 de mayo de 2019 suscripto por la entonces Secretaría de Gobierno de Salud, y la firma Biogen (Argentina). [consultado el 29.11.2020]. Disponible en:

https://www.boletinoficial.gob.ar/detalleAviso/primera/231312/20200629

30. Argentina. Administración Nacional de Alimentos, Medicamentos y Tecnología Médica. Disposición № 4529/20 dle 23 de junio de 2020. Dispone reinscribese el Certificado de inscripción en el Registro de Especialidades Medicinales № 58.924 correspondiente a la especialidad medicinal SPINRAZA/NUSINERSEN, solución para inyección intratecal, 12 $\mathrm{mg} / 5 \mathrm{ml}$, cuya titularidad corresponde a la firma BIOGEN ARGENTINA S.R.L. [consultado el 29.11.2020]. Disponible en:

http://www.anmat.gob.ar/boletin_anmat/Junio_2020/Dispo_4529-20.pdf

31. Argentina. Ministerio de Salud de la Nación. Resolución № 1115/2020 del 26 de junio de 2020. Derógase la Resolución № 1452 del 9 de agosto de 2019 de la entonces SECRETARÍA DE GOBIERNO DE SALUD. [consultado el 29.11.2020]. Disponible en: https://www.boletinoficial.gob.ar/detalleAviso/primera/231313/20200629

32. Argentina. Superintendencia de Servicios de Salud. Resolución № 597/2020 del 27 de junio de 2020. [consultado el 29.11.2020]. Disponible en:

https://www.boletinoficial.gob.ar/detalleAviso/primera/231446/20200630

33. Argentina. Constitución Nacional. 15 de diciembre de 1994. Senado y Cámara de Diputados de la Nación Argentina. Boletín Oficial 3 de enero de 1995.

34. Argentina. Ministerio de Desarrollo Productivo. Resolución № 202/20 del 30 de junio de 2020 por medio de la cual se estableció el precio máximo de Nusinersen. [consultado el 
24.11.2020]. Disponible en:

https://www.boletinoficial.gob.ar/detalleAviso/primera/231517/20200701

35. Argentina. Ministerio de Salud de la Nación. Resolución № 1860/2020 del 11 de noviembre de 2020 por medio de la cual se derogan las Resoluciones No 1453/2019 y Nº 1886/2019. [consultado el 29.11.2020]. Disponible en:

https://www.boletinoficial.gob.ar/detalleAviso/primera/237295/20201113

36. Brasil. Resolución RDC № 202/2018 del 8 de enero de 2018. Brasília: Diário Oficial da União, 2018 [consultado el 17.11.2020]; edición 5, sección 1, p. 3-6, 54. Disponible en: https://www.in.gov.br/materia/-/asset_publisher/Kujrw0TZC2Mb/content/id/1652079/do1 2018-01-08-resolucao-rdc-n-208-de-5-de-janeiro-de-2018-1652075

37. Brasil. Ministerio de Salud. Portaria № 24 de fecha 24 de abril de 2019, por la cual se publica la decisión de incorporar Nusinersen para el tratamiento de la atrofia muscular espinal (AME) 5q tipo I, en el ámbito del Sistema Único de Salud - SUS. [consultado con fecha 19.11. 2020]. Disponible en: https://www.in.gov.br/web/dou/-/portaria-no-24-de-24-deabril-de-2019-85049724

38. Brasil. Ministerio de Salud de Brasil. Portaria Conjunta № 15 del 22 de octubre de 2019. Aprova o Protocolo Clínico e Diretrizes Terapêuticas da Atrofia Muscular Espinhal 5q tipo I. [consultado el 29.11.2020]. Disponible en:

http://conitec.gov.br/images/Protocolos/Portaria_Conjunta_PCDT_Atrofia_Muscular_Espinh al_5q_Tipo-I.pdf

39. Brasil. Ministerio de Salud de Brasil. Portaria № 1297 del 11 de junio de 2019. Institui projeto piloto de acordo de compartilhamento de risco para incorporação de tecnologias em saúde, para oferecer acesso ao medicamento Spinraza (Nusinersena) para o tratamento da Atrofia Muscular Espinhal (AME 5q) tipos II e III no âmbito do Sistema Único de Saúde SUS. Brasília: Diário Oficial da União, 12 de junio de 2019 [consultado el 16.11.2020]; edição 112, seção 1, p. 125. Disponible en: https://www.in.gov.br/en/web/dou/-/portaria-n1.297-de-11-de-junho-de-2019-163114948

40. Ramos TM, Thomasi TZ, Duarte Júnior DP. Acordos de compartilhamento de riscos para aquisição do medicamento Spinraza® no Brasil: novas perspectivas sobre a proteção jurídica dos pacientes. Cad. Ibero Am. Direito Sanit. [Internet]. abr./jun. 2020 [consultado el 14.11.2020];9(2):99-115. Disponible en:

https://www.cadernos.prodisa.fiocruz.br/index.php/cadernos/article/view/656/738. doi: https://doi.org/10.17566/ciads.v9i2.656

41. Argentina. Ministerio de Desarrollo Productivo. Resolución № 202/20 del 30 de junio de 2020 por medio de la cual se estableció el precio máximo de Nusinersen. [consultado el 24.11.2020]. Disponible en:

https://www.boletinoficial.gob.ar/detalleAviso/primera/231517/20200701

42. Carignani A, Delduque MC. Ações judiciais argentinas em saúde e consequências legais na jurisdição brasileira. Cad. Ibero Am. Direito Sanit. [Internet]. 20 de dezembro de 2012 [consultado el 24.11.2020];1(2):72-86. Disponible en: 
https://www.cadernos.prodisa.fiocruz.br/index.php/cadernos/article/view/46. doi: https://doi.org/10.17566/ciads.v1i2.46

\section{Colaboradores}

Todos los autores contribuyeron en la redacción, investigación, interpretación de los datos y aprobó la versión final.

Como citar este artigo:

Carignani A, Flores DY. Estrategia de Estado para el tratamiento de la atrofia muscular espinal: un desafío del sistema de salud en Argentina. Cadernos Ibero-Americanos de Direito Sanitário. 2020 out./dez.; 9(4): 70-88.

https://doi.org/10.17566/ciads.v9i4.749 